\title{
Unruptured left ventricular pseudoaneurysm following inferior wall myocardial infarction
}

\author{
Laxman Dubey ${ }^{1}$, Rabindra Timala ${ }^{2}$, Ridhi Adhikari ${ }^{3}$, \\ Sanjib Sharma ${ }^{1}$, Mani Gautam ${ }^{1}$, Samir Gautam ${ }^{1}$ \\ ${ }^{1}$ Department of Cardiology, College of Medical Sciences and Teaching Hospital, Bharatpur, Nepal \\ ${ }^{2}$ Department of Cardiac Surgery, Shahid Gangalal National Heart Centre, Kathmandu, Nepal \\ ${ }^{3}$ Department of Radiodiagnosis, College of Medical Sciences and Teaching Hospital, Bharatpur, Nepal
}

\begin{abstract}
Left ventricular (LV) pseudoaneurysm is a rare but potentially lethal complication of acute myocardial infarction (MI). We report a very rare case of a 60 year-old woman with a ruptured myocardial wall, and a non-ruptured LV pseudoaneurysm. The patient presented with acutely worsening shortness of breath and exertional dyspnea of one month's duration, and palpitation. She had an inferior wall MI nine months previously. Coronary angiography showed severe stenosis at right coronary artery. Echocardiography, LV angiography, and computed tomography angiography revealed a large pseudoaneurysm postero-inferior to the $L V$. Surgical resection of the pseudoaneurysm was performed and repair of the ruptured $L V$ wall done, with good results. (Cardiol J 2012; 19, 5: 539-542)
\end{abstract}

Key words: left ventricular pseudoaneurysm, myocardial infarction, surgery

\section{Introduction}

Left ventricular (LV) pseudoaneurysm is a very rare complication following acute myocardial infarction (MI). LV pseudoaneurysm results from a rupture of the ventricular free wall. An organizing thrombus and hematoma, together with the pericardium, seal the rupture of the LV, leading to a localized hemopericardium and thus preventing cardiac tamponade. With time, this area of organized thrombus and pericardium can become a pseudoaneurysm [1].

Transmural MI is the commonest cause of LV pseudoaneurysm, but may result from cardiac surgery, trauma and infection [2]. Since untreated LV pseudoaneurysm has a $30 \%$ to $45 \%$ risk of rupture, early surgical intervention is recommended because there is a risk of rupture even with a small pseudoaneurysm [3].

Here, we describe a 60 year-old woman who had been diagnosed via transthoracic echocardio- graphy, cardiac catheterization, and computed tomography (CT) angiogram as having a pseudoaneurysm nine months after an inferior wall MI. She received surgical treatment for her pseudoaneurysm and had a good postoperative outcome.

\section{Case report}

A 60 year-old female presented to our institution with a complaint of dyspnea (NYHA II-III). She had a history of hypertension of three years' duration, sustained an inferior wall MI nine months ago and underwent conservative treatment at that time. Subsequently, she had been healthy, free of symptoms of heart failure, and was on follow up in the outpatient department on a regular basis. Nine months later, she presented with acutely worsening shortness of breath and exertional dyspnea of one month's duration, and palpitation. The patient's blood pressure was $110 / 70 \mathrm{~mm} \mathrm{Hg}$, pulse rate of

Address for correspondence: Dr. Laxman Dubey, Consultant Cardiologist, College of Medical Sciences and Teaching Hospital, Bharatpur, Nepal, tel: 00977-9851123288, e-mail: dubeylax@yahoo.com 


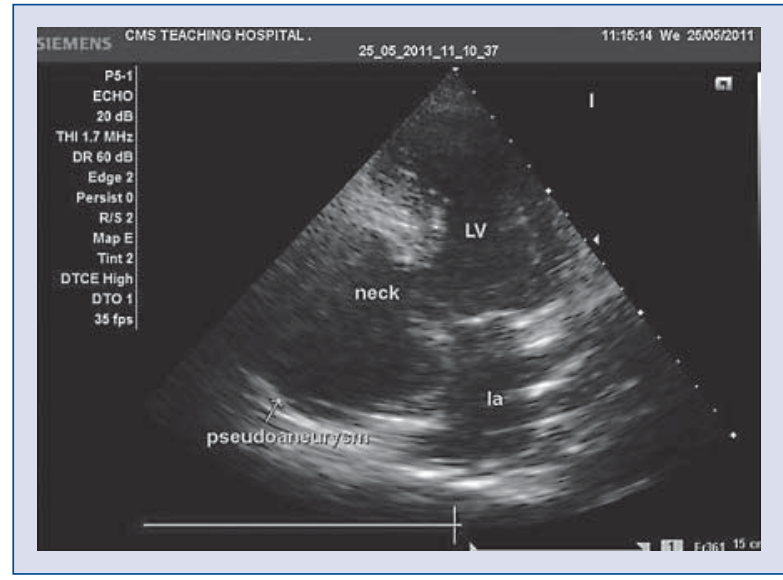

Figure 1. Transthoracic two-dimensional echocardiography shows a large, echo-free space behind the postero-inferior wall, which communicates with the left ventricle (LV) through a narrow neck.

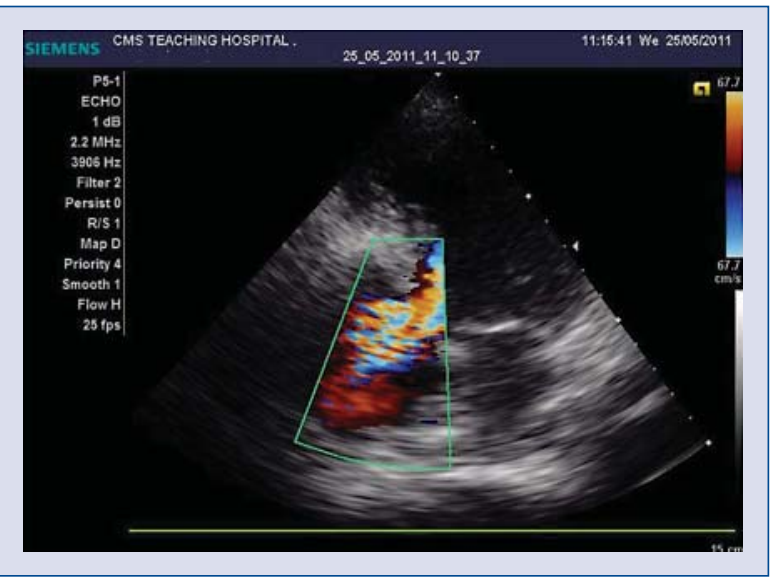

Figure 2. Color Doppler study demonstrates passage of blood from the left ventricular cavity into the pseudoaneurysm.

$85 \mathrm{bpm}$ which was regular and equal in both arms, and respiratory rate was 18 breaths/min. Examination of the neck did not reveal carotid bruit, but was positive for mildly elevated jugular venous pressure. Cardiac examination revealed normal heart sounds, with a grade III pansystolic murmur at the cardiac apex. Chest auscultation revealed fine bibasilar crackles.

A chest X-ray showed mild cardiomegaly, and an electrocardiogram showed sinus rhythm at $86 \mathrm{bpm}$, T-wave inversion and a $\mathrm{Q}$ wave in leads II, III, and aVF. Hemoglobin, white cell count, differential white cell count, serum electrolytes, renal function tests, liver function tests, and cardiac enzymes were within normal limits. Transthoracic echocardio-

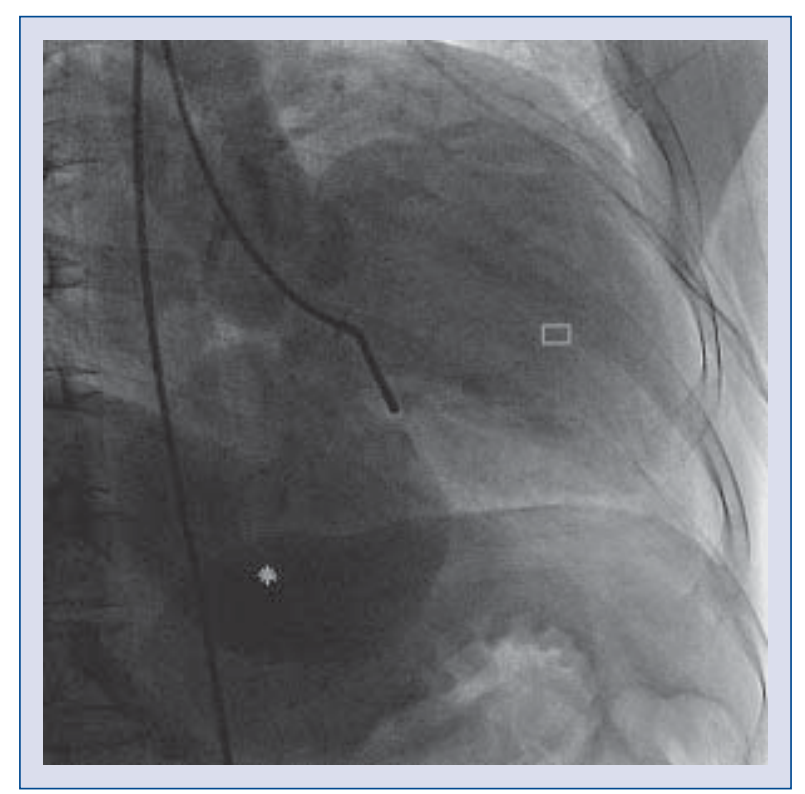

Figure 3. Left ventriculography reveals a postero-inferiorly positioned large aneurysm (asterisk) communicating with left ventricle (rectangle).

graphy showed the presence of a dyskinetic cavity connected to the postero-inferior wall that communicated freely with the LV; a pseudoaneurysm was suspected (Fig. 1).

Color Doppler study demonstrated passage of blood from the LV cavity into the pericardium through an opening in the LV (Fig. 2). Mild-to-moderate mitral regurgitation was noted in the color Doppler study.

The patient subsequently underwent diagnostic coronary angiography and left ventriculography. Coronary angiography revealed $80 \%$ stenosis at the proximal third and 95\% stenosis at the distal third of the right coronary artery. Left system was normal. Left ventriculography revealed a postero-inferiorly positioned large aneurysm, which communicated with the LV cavity through a relatively small orifice (Fig. 3). There was a grade III mitral regurgitation.

The patient was referred to the cardiac surgeon for possible coronary artery bypass graft with repair of the pseudoaneurysm. CT angiography was done which showed a large bulging sac-like lesion with a neck portion in the postero-inferior wall of the LV, which further confirmed the presence of a large pseudoaneurysm (Fig. 4). The patient underwent successful patch repair of the pseudoaneurysm (Figs. 5A, B). Her further hospital course was uneventful and she was subsequently discharged after seven days. 


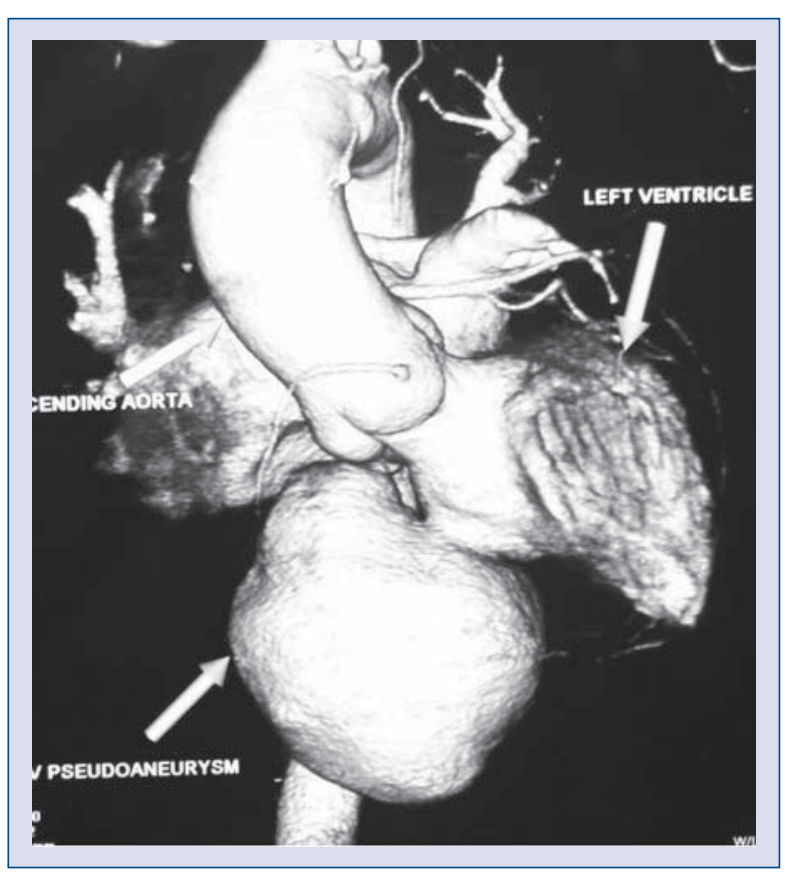

Figure 4. Computed tomography angiogram shows a focal, bulging, sac-like lesion attached to the postero-inferior wall of left ventricle.

\section{Discussion}

Rupture of the LV free wall is a catastrophic complication occurring in $4 \%$ of patients after acute MI, and in $23 \%$ of those who die of MI [4]. However, $\mathrm{LV}$ pseudoaneurysm is a result of a rupture of the ventricular free wall, contained by overlying adherent pericardium or scar tissue. Therefore, the walls of pseudoaneurysms are composed of organized hematoma and pericardium and lack any elements of the original myocardial wall. LV pseudoaneurysm is, thus, rare because in most instances ventricular free-wall rupture leads to pericardial tamponade and sudden death.

LV pseudoaneurysm is characterized by the nature of their walls, neck, and risk of rupture. Acute MI is the commonest cause of LV pseudoaneurysm, followed by cardiac surgery, trauma, and infection [2]. On the other hand, LV true aneurysm is most commonly the result of MI and is defined as areas of thinned myocardium which are dyskinetic and involve the full thickness of the wall [5].

LV pseudoaneurysms typically have a neck narrower than the diameter of the LV true aneurysm and are more often located in the postero-inferior wall than the anterior wall. This is in contrast to LV true aneurysms, which are more often seen in the anterior wall and apex, and have a wide neck [5]. Differentiating a LV pseudoaneurysm from a LV true aneurysm is important because of the different methods of treatment recommended. LV pseudoaneurysm has a greater tendency to rupture compared to LV true aneurysm [6], but there are occasional reports of prolonged survival of patients with an unruptured LV pseudoaneurysm [7], as in our case. Although the risk of rupture is high, patients with an unruptured LV pseudoaneurysm usually present with congestive heart failure, arrhythmia and embolism. Diagnosing LV pseudoaneurysm is difficult: nearly $10 \%$ of patients can be asymptomatic [2], and some patients may have non-specific complaints such as cough, altered mental status, and

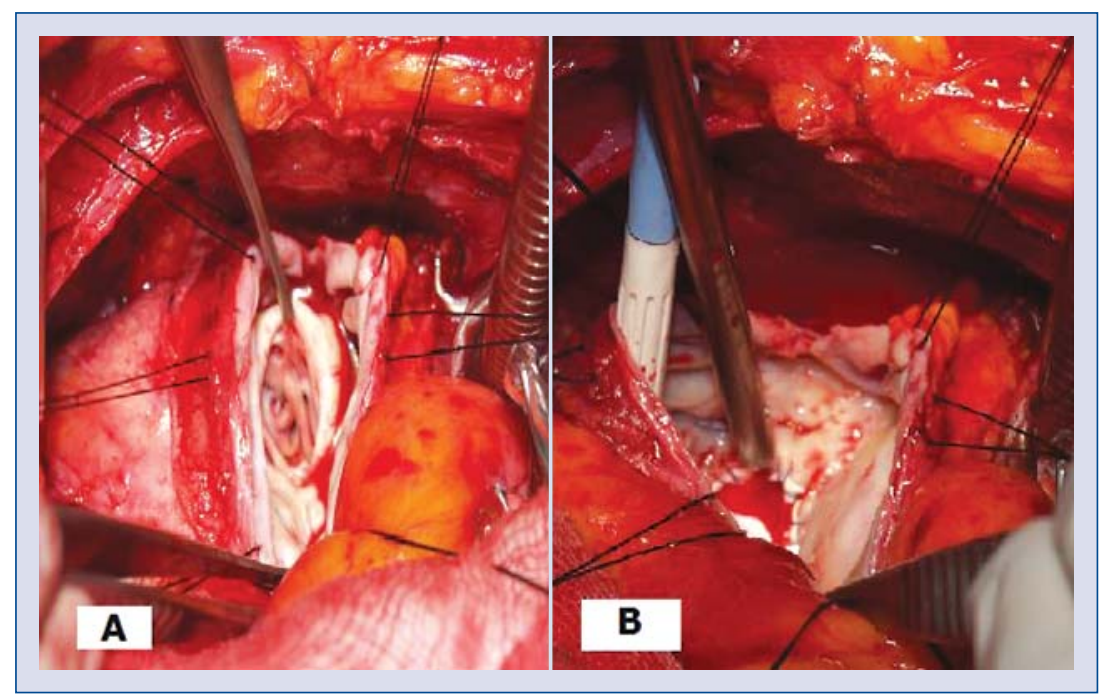

Figure 5. A. The pseudoaneurysm is opened; B. Patch closure of the defect. 
dizziness that rarely elicit a concern for a LV pseudoaneurysm [2]. The commonest clinical presentation of pseudoaneurysm is characterized by congestive heart failure (36\%), chest pain (30\%), and dyspnea (25\%), whereas the incidence of sudden death as a presenting symptom is $3 \%$ [8].

The diagnosis of pseudoaneurysm is usually suggested by imaging modalities such as transthoracic/transesophageal echocardiography, LV angiography, magnetic resonance imaging (MRI), CT and radionuclide scanning [9].

Echocardiography is a valuable non-invasive test for diagnosing pseudoaneurysms. Echocardiography before surgery is useful in differentiating a LV aneurysm from a LV pseudoaneurysm. Echocardiography can be used to compare the diameter of the neck of the aneurysm with its maximum diameter. It has been found that the ratio of the maximum diameter of the orifice to the maximum internal diameter of the cavity is between 0.25 and 0.50 for pseudoaneurysms, while for true aneurysms it is between 0.90 and 1.0 [10]. The presence of turbulent flow by color Doppler at the neck of a cavity or within the cavity itself also suggests the presence of a pseudoaneurysm [11].

Recent reports have indicated that cardiac multi-slice CT is a sensitive technique for detecting a LV pseudoaneurysm [12]. Moreover, cardiac MRI may represent an effective diagnostic tool, as cardiac MRI allows visualization of the entire heart and is able to clearly distinguish between structures such as pericardium, myocardium thrombus and epicardial fat [13].

Ventricular free wall rupture occurs in most cases of cardiac rupture and is usually associated with sudden cardiac death because of hemopericardium and subsequent cardiac tamponade [14].

It is generally accepted that high mortality rates exist for patients with LV pseudoaneurysm who do not undergo surgery. Therefore, surgical resection is recommended by most authors as soon as a LV pseudoaneurysm is diagnosed because of the risk of rupture. However, some authors believe that medical treatment of chronic LV pseudoaneurysm of more than three months is not associated with an increased risk for cardiac rupture, and that surgical resection should be considered in patients with ventricular tachycardia, recurrent embolism detected within three months after MI, or who develop congestive heart failure related to a LV pseudoaneurysm [15]. In our patient, surgical treatment was chosen because of the unusually large size of the LV pseudoaneurysm and the presence of signs of congestive heart failure which was thought to be related to the LV pseudoaneurysm.

\section{Conclusions}

Rupture of the LV free wall is a catastrophic complication occurring in $4 \%$ of patients after MI; however, LV pseudoaneurysm is a result of a rupture of the LV free wall contained by overlying adherent pericardium or scar tissue. The diagnosis of a LV pseudoaneurysm requires a high index of suspicion due to the often atypical symptomatology. The early diagnosis of LV pseudoaneurysm is essential to avoid complications and to determine appropriate treatment. Once diagnosed, LV pseudoaneurysm should be operated upon urgently due to the high risk of rupture.

\section{Conflict of interest: none declared}

\section{References}

1. Dachman AH, Spindola-Franco H, Solomon N. Left ventricular pseudoaneurysm: Its recognition and significance. JAMA, 1981; 246: 1951-1953.

2. Frances C, Romero A, Grady D. Left ventricular pseudoaneurysm. J Am Coll Cardiol, 1998; 32: 557-561.

3. Catherwood E, Mintz GS, Kotler MN et al. Two-dimensional echocardiographic recognition of left ventricular pseudoaneurysm. Circulation, 1980; 62: 294-303.

4. Pollak H, Nobis H, Miczoc J. Frequency of left ventricular free wall ruptures complicating acute myocardial infarction since the advent of thrombolysis. Am J Cardiol, 1994; 74: 184-186.

5. Brown SL, Gropler RJ, Harris KM. Distinguishing left ventricular aneurysm from pseudoaneurysm: A review of the literature. Chest, 1997; 111: 1403-1409.

6. Vlodaver Z, Coe JI, Edwards JE. True and false left ventricular aneurysms: Propensity for the latter to rupture. Circulation, 1975; 51: 567-572.

7. Hung MJ, Wang $\mathrm{CH}$, Chang WJ. Unruptured left ventricular pseudoaneurysm following myocardial infarction. Heart, 1998; 80: 94-97.

8. Contuzzi R, Gatto L, Patti G et al. Giant left ventricular pseudoaneurysm complicating an acute myocardial infarction in patient with previous cardiac surgery: A case report. J Cardiovasc Med (Hagerstown), 2009; 10: 81-84.

9. Kocak H, Becit N, Ceviz M, Unlu Y. Left ventricular pseudoaneurysm after myocardial infarction. Heart Vessels, 2003; 18: 160-162.

10. Gatewood RP, Nanda NC. Differentiation of left ventricular pseudoaneurysm from true aneurysm with two-dimensional echocardiography. Am J Cardiol, 1980; 46: 869-878.

11. Loperfido F, Pennestri F, Mazzari M et al. Diagnosis of left ventricular pseudoaneurysm by pulsed Doppler echocardiography. Am Heart J, 1985; 110: 1291-1293.

12. Niimura H, Mito T, Matsunaga A et al. Left ventricular pseudoaneurysm following acute myocardial infarction. Intern Med, 2006; 45: 1221-1223.

13. Konen E, Merchant N, Gutierrez C et al. True versus false left ventricular aneurysm: Differentiation with MR imaging. Initial experience. Radiology, 2005; 236: 65-70.

14. Bates RJ, Beutler S, Resnekov L et al. Cardiac rupture challenge in diagnosis and management. Am J Cardiol, 1977; 40: 429-437.

15. Natarajan MK, Salerno TA, Burke B, Chiu B, Armstrong PW Chronic false aneurysms of the left ventricle: Management revisited. Can J Cardiol, 1994; 10: 927-931. 